\title{
Assessing Accuracy of an Analytical Method in silico: Application to "Accurate Constant via Transient Incomplete Separation" (ACTIS)
}

\author{
Jean-Luc Rukundo ${ }^{1}$, J.C. Yves Le Blanc ${ }^{2}$, Sven Kochmann ${ }^{1}$, Sergey N. Krylov ${ }^{1 *}$ \\ ${ }^{1}$ Department of Chemistry and Centre for Research on Biomolecular Interactions, York University, Toronto, Ontario M3J 1P3, Canada \\ ${ }^{2}$ SCIEX, Vaughan, Ontario L4K 4V8, Canada.
}

Supporting information placeholder

ABSTRACT: Analytical methods may not have reference standards required for testing their accuracy. We postulate that accuracy of an analytical method can be assessed in the absence of reference standards in silico if the method is built upon deterministic processes. A deterministic process can be precisely computer-simulated thus allowing virtual experiments with virtual reference standards. Here, we apply this in silico approach to study "Accurate Constant via Transient Incomplete Separation" (ACTIS), a method for finding the equilibrium dissociation constant $\left(K_{\mathrm{d}}\right)$ of protein-small molecule complexes. ACTIS is based on a deterministic process: molecular diffusion of the interacting protein-small molecule pair in a laminar pipe flow. We used COMSOL software to construct a virtual ACTIS setup with a fluidic system mimicking that of a physical ACTIS instrument. Virtual ACTIS experiments performed with virtual samples mixtures of a protein and a small molecule with defined rate constants and, thus, $K_{\mathrm{d}}$ of their interaction - allowed us to assess ACTIS accuracy by comparing the determined $K_{\mathrm{d}}$ value to the input $K_{\mathrm{d}}$ value. Further, the influence of multiple system parameters on ACTIS accuracy was investigated. Within multi-fold ranges of parameters, the values of $K_{\mathrm{d}}$ did not deviate from the input $K_{\mathrm{d}}$ values by more than a factor of 1.25 strongly suggesting that ACTIS is intrinsically accurate and that its accuracy is robust. Accordingly, further development of ACTIS can focus on achieving high reproducibility and precision. We foresee that in silico accuracy assessment, demonstrated here with ACTIS, will be applicable to other analytical methods built upon deterministic processes.

Accuracy is one of the key performance parameters of quantitative analytical methods. Many methods do not have reference standards and, thus, their accuracy cannot be experimentally tested. We suggest that accuracy of an analytical method can be assessed in the absence of reference standards in silico if the underlying physicochemical processes are deterministic, i.e. can be precisely simulated. Detailed computer simulation should facilitate virtual experiments in which a virtual sample can be perfectly defined and, thus, can serve as a virtual reference standard. In this work, we applied this in silico accuracy assessment to "Accurate Constant via Transient Incomplete Separation (ACTIS)".

ACTIS is a separation-based method for finding $K_{\mathrm{d}}$ of complexes (PL) between proteins (P) and small-molecule ligands $(\mathrm{L}):^{1}$

$$
\mathrm{P}+\mathrm{L} \underset{k_{\text {off }}}{\stackrel{k_{\text {on }}}{\rightleftarrows}} \mathrm{PL}
$$

where $K_{\mathrm{d}}$ is defined via equilibrium concentrations of $\mathrm{P}, \mathrm{L}$, and PL:

$$
K_{\mathrm{d}}=[\mathrm{L}]_{\mathrm{eq}}[\mathrm{P}]_{\mathrm{eq}} /[\mathrm{PL}]_{\mathrm{eq}}
$$

There is no reference standard for the determination of $K_{\mathrm{d}}$, i.e. there is no PL with a known $K_{\mathrm{d}}$, thus, accuracy of ACTIS cannot be assessed experimentally. On the other hand, ACTIS is built upon a deterministic process of molecular diffusion of $\mathrm{P}, \mathrm{L}$, and $\mathrm{PL}$, interacting as in eq 1 while moving within a laminar pipe flow. This process that can be precisely described by a set of partial differential equations with precisely defined initial and boundary conditions and precisely defined input values of the rate

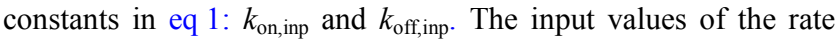
constants define the input value of the equilibrium constants:

$$
K_{\mathrm{d}, \text { inp }}=k_{\text {off,inp }} / k_{\text {on,inp }}
$$

which can serve as a virtual reference standard in virtual ACTIS experiments. Accordingly, ACTIS is suitable for in silico accuracy assessment.

In ACTIS, a short plug of an equilibrium mixture of $\mathrm{P}$ and $\mathrm{L}$ in a buffer solution is injected into a capillary pre-filled with the pure buffer solution. The plug is then propagated inside the capillary by a pressure-driven flow of the buffer solution. Different rates of transverse diffusion of PL and $\mathrm{L}$ in laminar flow cause their transient incomplete separation (TIS) (Figure 1A) resulting in a non-diffusive peak for PL and a diffusive peak for $\mathrm{L}$ (Figure 1B). To determine $K_{\mathrm{d}}$, TIS is performed for a series of
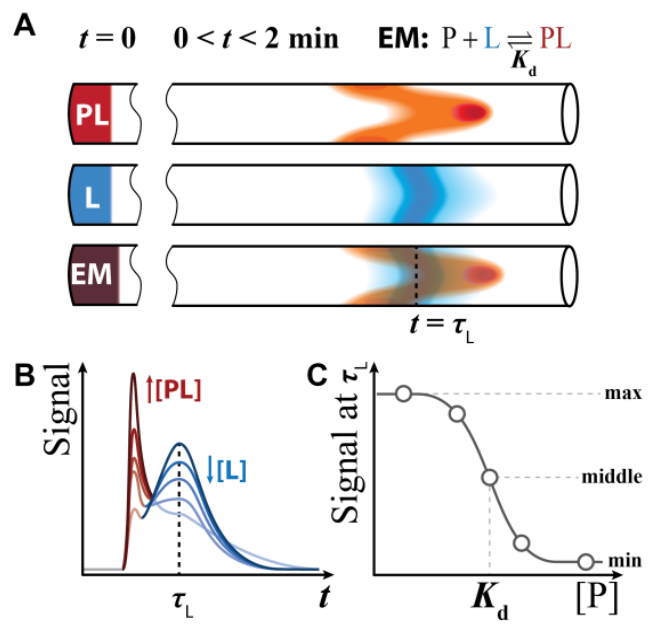

Figure 1. Simplified schematic of determining $K_{\mathrm{d}}$ by ACTIS. A: A short plug of the equilibrium mixture (EM) of $\mathrm{P}$ and $\mathrm{L}$ is propagated through a capillary. Differences in transverse diffusion of PL and L cause their longitudinal separation. B: Longitudinal separation results in two peaks, and a cumulative signal from $\mathrm{L}$ and $\mathrm{PL}$ is measured at time $\tau_{\mathrm{L}}$, which is the characteristic time of transverse diffusion of $\mathrm{L}$. The signal is measured at a constant concentration of $\mathrm{L}$ and varying concentrations of $\mathrm{P}$. C: A binding isotherm "signal-at- $\tau_{\mathrm{L}} v s$ concentration of $\mathrm{P}$ " is built, and $K_{\mathrm{d}}$ is found as the concentration of $\mathrm{P}$, which corresponds to the signal in the middle between the maximum and minimum signals.

equilibrium mixtures with a constant concentration of $\mathrm{L}$ and varying concentration of $\mathrm{P}$ producing a set of curves termed separagrams (Figure 1B). The cumulative signal of protein-bound $\mathrm{L}$ and unbound $\mathrm{L}$ is taken at the time corresponding to the maximum of the diffusive peak for each curve. Subsequently, a classical binding isotherm "signals $v s$ concentration of $\mathrm{P}$ " is built to reveal the value of $K_{\mathrm{d}}$ (Figure 1C). ACTIS is a uniquely deterministic method as it relies on molecular diffusion in a pressure-driven flow which can be described by a system of partial differential equations with fully-defined initial and boundary conditions. ${ }^{1-3}$ As such, it is perfectly suited for computational assessment of its accuracy, which does not appear to have any inherent sources of inaccuracy. 


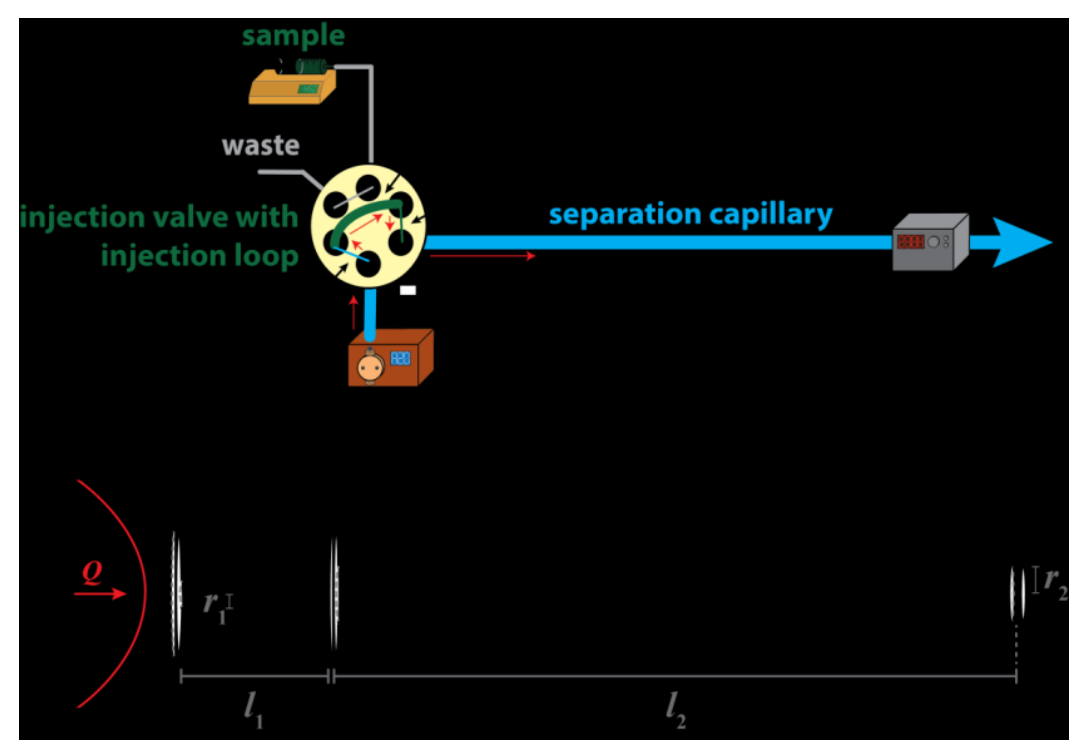

Figure 2. Schematics of a physical (A) and computational (B) ACTIS instrument. The five letters label the five components simulated in this study and their respective physical counterparts with default dimensions: pump tube (T, 5 -cm length, $400-\mu \mathrm{m}$ radius), injection loop $\left(\mathbf{I}, l_{1}=13 \mathrm{~cm}, r_{1}=50 \mu \mathrm{m}\right)$, separation capillary $\left(\mathbf{S}, l_{2}=50 \mathrm{~cm}, r_{2}=100 \mu \mathrm{m}\right)$ with a detection window (5-mm length, $100-\mu \mathrm{m}$ radius) at the end, and two identical connectors $(\mathbf{C}, 1.1$ mm length, 220- $\mu \mathrm{m}$ radius) linking $\mathbf{T}$ to $\mathbf{I}$ and $\mathbf{I}$ to $\mathbf{S}$. $Q$ is the volumetric flow rate provided by a virtual pump. Flow rates were different during sample injection from the loop into the capillary $\left(Q_{\text {inj }}=5 \mu \mathrm{L} / \mathrm{min}\right)$ and during the following TIS stage $\left(Q_{\mathrm{TIS}}=50 \mu \mathrm{L} / \mathrm{min}\right)$. $Q_{\text {inj }}$ and $Q_{\text {TIS }}$ were not varied in this study. By default, switch between $Q_{\text {inj }}$ and $Q_{\mathrm{TIS}}$ was set to $10 \mathrm{~ms}$.

A physical ACTIS instrument has a minimum fluidic system with a pump, an injection loop, a separation capillary, and a multiport valve (Figure $2 \mathrm{~A}) .^{1-3}$ The valve serves as two connectors: one from a pump tube to the injection loop and the other one from the injection loop to the separation capillary. Accordingly, the instrument has five essential fluidic components which can be presented as a series of coaxial pipes of different radii (Figure 2B). We used COMSOL to construct a five-component ACTIS setup depicted in Figure 2B with dimensions identical to those of a physical ACTIS instrument (described in the figure legend). We found that $K_{\mathrm{d}}$ determined in this setup deviated from $K_{\text {d,inp }}$, defined by eq 3 and used as a virtual reference standard, by a factor of 1.02. This setup was utilized to study how the accuracy of $K_{\mathrm{d}}$ was affected by variations in: (i) the radius of the injection loop while keeping its volume constant, (ii) the radius of the separation capillary, (iii) shape of the initial (prior to start of TIS) plug of the equilibrium mixture, and (iv) ramp time in flow-rate onset (after the start of TIS). The variations of parameters used in this study exceeded markedly the ones that are expected in a physical ACTIS setup. We found that despite multi-fold variations in a number of parameters, the maximum deviation of $K_{\mathrm{d}}$ from

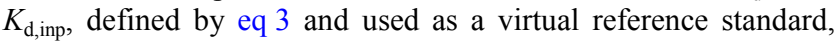
was less than a factor of 1.25 , suggesting robust intrinsic accuracy of ACTIS.

The intrinsic accuracy of ACTIS has a great practical importance as it allows ACTIS developers to vary instrument configuration without raising concerns about the influence of such variations on accuracy of $K_{\mathrm{d}}$. Efforts and resources can thus be focused on optimizing instrument configuration to achieve the best reproducibility and the highest precision, the second most important performance parameter of this quantitative analytical method.

\section{THEORETICAL BACKGROUND}

Here we describe theoretical aspects of ACTIS that are essential for understanding this method at the conceptual level and help establish the theoretical background required for understanding our current work. In particular, we explain basic principles of $K_{\mathrm{d}}$ determination and TIS of L from PL; the corresponding two sections are a close reiteration of our previously published explanation. ${ }^{1}$

General Procedure of $K_{\mathrm{d}}$ Determination. In general, finding $K_{\mathrm{d}}$ requires determination of a fraction $R$ of unbound $\mathrm{L}$ in the equilibrium mixture of $\mathrm{L}$ and $\mathrm{P}$ with the initial concentrations $[\mathrm{L}]_{0}$ and $[\mathrm{P}]_{0}$, respectively:

$$
R=[\mathrm{L}]_{\mathrm{eq}} /[\mathrm{L}]_{0}
$$

Finding $R$, in turn, requires a signal $S$ that is a superposition of signals from $\mathrm{L}$ and $\mathrm{PL}$ :

$$
S=S_{\mathrm{L}} \times R+S_{\mathrm{PL}} \times(1-R), S_{\mathrm{L}} \neq S_{\mathrm{PL}}
$$

where $S_{\mathrm{L}}$ and $S_{\mathrm{PL}}$ are the signals of pure L $\left([\mathrm{L}]=[\mathrm{L}]_{0},[\mathrm{PL}]=0\right)$ and pure PL $\left([\mathrm{L}]=0,[\mathrm{PL}]=[\mathrm{L}]_{0}\right)$, respectively; $S_{\mathrm{L}}$ and $S_{\mathrm{PL}}$ can be found in experiments with $[\mathrm{P}]_{0}=0$ and $[\mathrm{L}]_{0}<<[\mathrm{P}]_{0} \gg>K_{\mathrm{d}}$, respectively. The signal from pure $\mathrm{P}$ must be negligible with respect to $S$ shown in eq 5 even for $[\mathrm{L}]_{0}<<[\mathrm{P}]_{0}>>K_{\mathrm{d}}$.

From eq 5, $R$ can be experimentally determined via measuring three signals, $S_{\mathrm{L}}, S_{\mathrm{PL}}$, and $S$, and using the following expression:

$$
R_{\exp }=\left(S-S_{\mathrm{PL}}\right) /\left(S_{\mathrm{L}}-S_{\mathrm{PL}}\right)
$$

$R$ can be also expressed theoretically as a function of $K_{\mathrm{d}},[\mathrm{P}]_{0}$, and $[\mathrm{L}]_{0}:{ }^{1,4}$

$$
R_{\text {theor }}=-\frac{K_{\mathrm{d}}+[\mathrm{P}]_{0}-[\mathrm{L}]_{0}}{2[\mathrm{~L}]_{0}}+\sqrt{\left(\frac{K_{\mathrm{d}}+[\mathrm{P}]_{0}-[\mathrm{L}]_{0}}{2[\mathrm{~L}]_{0}}\right)^{2}+}
$$

Then, a standard way of finding $K_{\mathrm{d}}$ is to determine $R_{\text {exp }}$ for a wide range of $[\mathrm{P}]_{0}$ at a constant $[\mathrm{L}]_{0}$ and to plot a binding isotherm: $R_{\exp }$ $v s[\mathrm{P}]_{0}$. Finally, this binding isotherm is fitted with eq 7 using $K_{\mathrm{d}}$ as a fitting parameter and the best fit reveals the sought value of $K_{\mathrm{d}}{ }^{1}$

Fundamentally, finding $R_{\exp }$ with eq 6 requires that $S_{\mathrm{L}}$ and $S_{\mathrm{PL}}$ be measurable and that $S_{\mathrm{L}} \neq S_{\mathrm{PL}}$. The latter inequality of signals from pure L and pure PL requires that L and PL be "separated" either spectrally or physically. ${ }^{5}$ Importantly, complete separation is not required if a signal from the mixture of $\mathrm{L}$ and $\mathrm{PL}$ is a superposition of signals from individual components $\mathrm{L}$ and PL comprising the mixture (see eq 5), and if pure $\mathrm{P}$ does not 
contribute to the cumulative signal from L and PL. As a result, spectral methods that provide only incomplete separation of signals from L and PL (optical spectra of L and LP do overlap typically) are common in finding $R_{\text {exp. }}{ }^{5-7}$ ACTIS facilitates finding $R_{\text {exp }}$ via incomplete physical separation of L and PL.

Transient Incomplete Separation of L from PL. TIS of L from PL will occur always when a short plug of their mixture is propagated within a Hagen-Poiseuille laminar flow in a long capillary. Such a flow is established by a pressure difference between the capillary ends and has a characteristic parabolic profile of flow velocity: the velocity ranges from zero at the capillary walls to its maximum in the capillary center. ${ }^{8}$ TIS of $\mathrm{L}$ from PL in the longitudinal direction is possible due to the difference in rates of transverse diffusion between small-size $\mathrm{L}$ and large-size PL. PL that is near the capillary center will diffuse to the capillary wall slower than L and, thus, will be displaced longitudinally by the flow more than L. PL located near the capillary wall will diffuse to the capillary center slower than L and will be displaced longitudinally by the flow less than L. As a result, during a short transitional stage, a bulk of PL moves faster than a bulk of L, while a tail of PL moves slower than that of L. The separation is incomplete, i.e. the longitudinal concentration profiles (concentration $v s$ position in the capillary) of L and PL do overlap, even during the transitional stage. Further, this separation gradually dissipates, i.e. the longitudinal concentration profiles of $\mathrm{L}$ and PL become symmetrical around the same symmetry axis, after the transitional stage. The past-TIS stage is described by the well-known Taylor dispersion. ${ }^{9}$

Tracking TIS is viewed to be optimal with a flow system in which the optimum distance from the starting position of the equilibrium-mixture plug to the detector $\left(l_{\text {opt }}\right)$ is linked with the average flow velocity $\left(v_{\mathrm{av}}\right)$, characteristic time of transverse diffusion of $\mathrm{L}$ from the center of the capillary to its inner wall $\left(\tau_{\mathrm{L}}\right)$, the volumetric flow rate $(Q)$, and diffusion coefficient of $\mathrm{L}$ $\left(\mu_{\mathrm{L}}\right)$, as follows: ${ }^{1,10}$

$$
l_{\mathrm{opt}}=v_{\mathrm{av}} \tau_{\mathrm{L}}=Q /\left(\pi \mu_{\mathrm{L}}\right)
$$

where $v_{\mathrm{av}}$ relates to $Q$ and the inner capillary radius $(r)$ as:

$$
v_{\mathrm{av}}=Q /\left(\pi r^{2}\right)
$$

The characteristic time of transverse diffusion across the capillary is defined in general as:

$$
\tau=r^{2} / \mu
$$

Longitudinal concentration profiles of $\mathrm{L}$ and $\mathrm{PL}$ are partially separated in the time domain as shown in Figure 1B. Further, if a signal can be measured for each of L and PL, with the abovementioned detector, inside the capillary or at its exit and is proportional to the average cross-sectional concentration of each of them, $S_{\mathrm{L}} \propto[\mathrm{L}]$ and $S_{\mathrm{PL}} \propto[\mathrm{PL}]$, then, the cumulative signal $S$ satisfies eq. 5 and can, thus, be used to determine $R_{\exp }$ with eq. 6 . Finally, $R_{\text {exp }}$ can be measured for a wide range of $[\mathrm{P}]_{0}$ to construct a classical binding isotherm $R_{\exp } v s[\mathrm{P}]_{0}$, which, in turn, can be used to find $K_{\mathrm{d}}$ via fitting the isotherm with eq. 7 .

\section{DESIGNING A VIRTUAL ACTIS EXPERIMENT}

Five-Component Virtual ACTIS Setup. The five-component physical ACTIS system depicted in Figure 2A operates as follows. The injection loop is filled with the sample. The sample is slowly transferred into the separation capillary at a distance equal to the sample-plug length from the capillary entry. The sample is then propagated fast through the separation capillary to cause TIS of L from PL.

We used COMSOL to construct a virtual five-component ACTIS setup with default dimensions identical to those of a physical ACTIS instrument (Figure 2B). The dimensions of the two connectors are identical and can be considered fixed as well as the dimensions of the pump tube. The dimensions of the injection loop and the separation capillary may need to be changed and, thus, were varied in this study. Of course, the virtual system also contains representations of a virtual pump (a source of a hydrodynamic flow characterized by its volumetric flow rate $Q$ ) and a virtual detector (a detection volume inside the capillary), which are also associated with some parameters that can vary and potentially cause inaccuracy in $K_{\mathrm{d}}$.

A default initial configuration in the virtual five-component setup is the one in which the loop and the loop-capillary connector are filled with the sample, while the remaining components are filled with the buffer solution. This situation mirrors the physical ACTIS setup. In a default virtual ACTIS experiment, the sample is slowly transferred from the injection loop to the separation capillary $\left(Q=Q_{\text {inj }}\right)$ and then propagated fast inside the capillary as in the physical ACTIS experiment $\left(Q=Q_{\text {TIS }}\right)$.

Further in this work, the five-component setup was used first to test the accuracy of $K_{\mathrm{d}}$ for the default geometry (Figure 2B). Then, in a more rigorous study, it was used to investigate how the accuracy of $K_{\mathrm{d}}$ determined with ACTIS was affected by variations in: (i) the radius of the injection loop while keeping its volume constant, (ii) the radius of the separation capillary, (iii) shape of the initial (prior to start of TIS) plug of the equilibrium mixture, and (iv) ramp time in flow-rate onset (after the start of TIS). Below, we provide details on how these four parts of the study were set up.

Geometry of the Injection Loop and Separation Capillary. There are four parameters that characterize the geometry of the injection loop and the separation capillary: the radius $\left(r_{1}\right)$ and length $\left(l_{1}\right)$ of the injection loop and the radius $\left(r_{2}\right)$ of the separation capillary and the distance from the beginning of the separation capillary to the centre of the virtual detector $\left(l_{2}\right)$. We conducted two sets of virtual ACTIS experiments in which $r_{1}$ and $r_{2}$ were varied. In one set of virtual experiments, $r_{1}$ was varied from $5 \mu \mathrm{m}$ to $5000 \mu \mathrm{m}$ ( $l_{1}$ was changed accordingly to keep the volume of the injection loop constant), while $r_{2}$ and $l_{2}$ were kept constant at default values linked through eqs $8-10$. In the other set of virtual experiments, $r_{2}$ was varied from $5 \mu \mathrm{m}$ to $5000 \mu \mathrm{m}$ while holding constant $r_{1}, l_{1}$, and $l_{2}$. The rest of the parameters were default (see Figure 2B).

Geometry of the Initial Sample Plug. While the ideal shape of the initial plug of the equilibrium mixture in the beginning of the separation capillary is cylindrical, in a real experiment, it will be distorted to some degree due to the imperfections of the injection process. To assess how such distortions can affect the accuracy of $K_{\mathrm{d}}$, we examined square, Gaussian, and half-Gaussian longitudinal distributions of concentrations of $\mathrm{L}, \mathrm{PL}$, and $\mathrm{P}$ in the initial plug (Figure 3A); cross-sectional concentration profiles were uniform. Here, as opposed to a default way of injecting a $3.0 \mathrm{~cm}$-long sample plug from the injection loop, the same-length initial plug was defined near the entrance of the separation capillary at a distance equal to the plug length, and no injection process was simulated. To model this initial plug shape, the longitudinal (along axis $x$ ) distribution of concentrations was defined as a square wave function:

$$
f\left(k_{1}, k_{2}, x\right)=H\left(k_{1} x / L\right)-H\left(k_{2} x / L-1\right)
$$

where $H(k x)=1 /\left(1+e^{-2 k x}\right)$, which is an analytical sigmoidal approximation of the Heaviside step function. ${ }^{11}$ The shape of the plug was varied by varying both $k_{1}$ and $k_{2}$. The condition of $k_{1}=k_{2}$ corresponds to symmetric plug shapes, e.g. for $k_{1}=k_{2}=2$ the plug shape is Gaussian and for $k_{1}=k_{2}=200$ the plug shape is a close approximation of the square function. For $k_{1} \neq k_{2}$ the plug shape is asymmetric, e.g. half-Gaussians. The rest of the parameters were default (see Figure 2B).

Ramp Time in Flow-Rate Onset. Achieving and maintaining the aforementioned ideal plug shape requires the injection of a sample plug from the injection loop into the separation capillary at a slow flow rate of $Q_{\text {inj }}$ for a time period of $t_{\text {inj. }}$. TIS, on the other hand, requires the propagation of the injected sample plug though the separation capillary at a high flow rate of $Q_{\text {TIS. }}$ In a virtual 

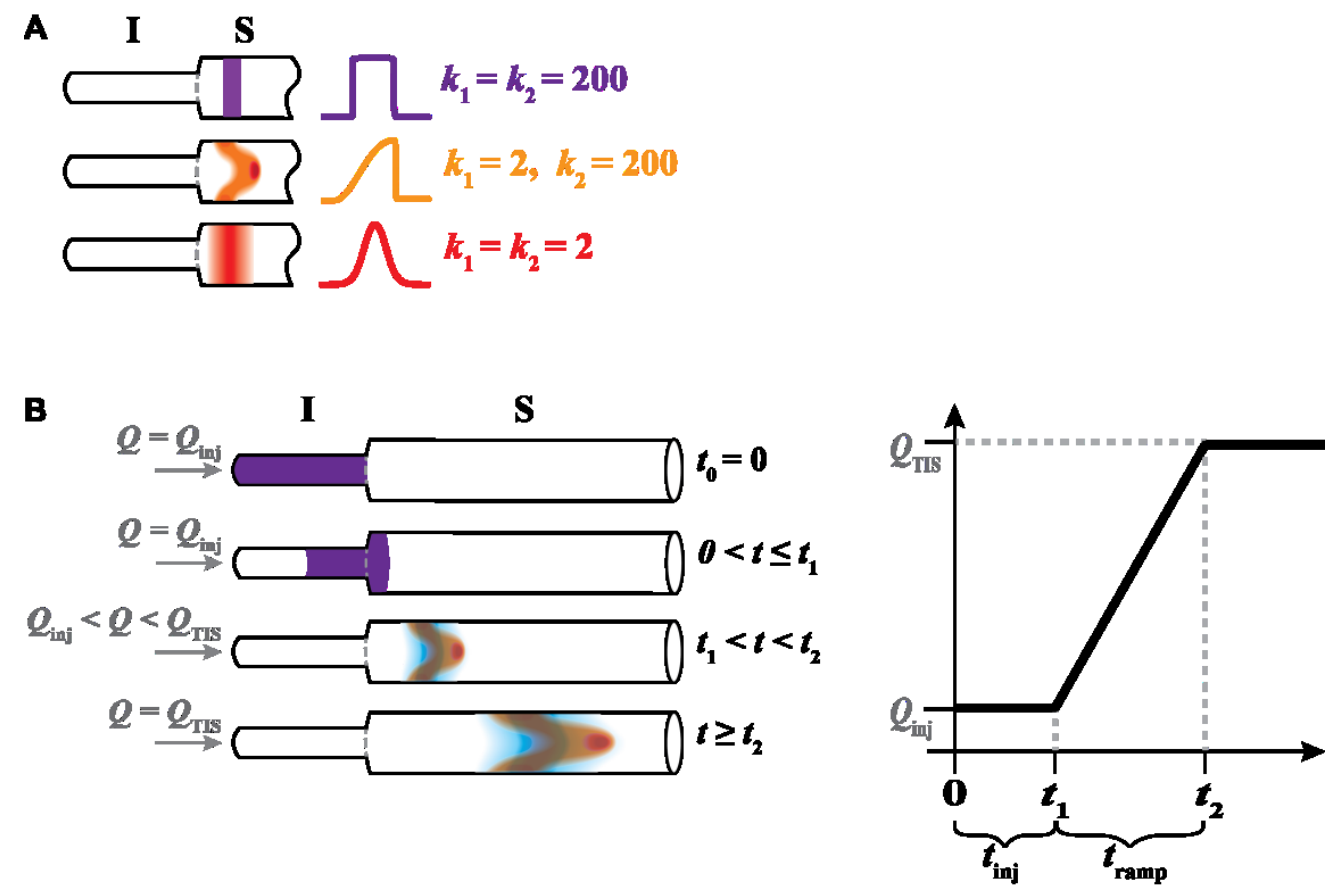

Figure 3. Schematic of the simulated geometries in ACTIS. For clarity the pump tube (T) and connectors (C) are omitted in the presentations here. A: A schematic of three different initial plug shapes at the entrance of the separation capillary. Different concentration distributions inside the capillary (left) and longitudinal concentration profiles (result of integration across the capillary, middle) are obtained by changing parameters $k_{1}$ and $k_{2}$ (right) in the Heaviside function. B: A schematic showing three steps in the injection of the mixture into the separation capillary and its propagation inside the capillary. The sample plug is injected from the injection loop into the separation capillary for a time period of $t_{\text {inj }}$ at a flow rate of $Q_{\text {inj. }}$. Immediately after completing the injection, the volumetric flow rate is set to $Q_{\text {TIS }}$ and the plug is further propagated through the separation capillary. The time interval required to reach the propagation flow rate $Q_{\text {TIS }}$ is $t_{\text {ramp }}$.

ACTIS experiment, the transition from $Q_{\text {inj }}$ to $Q_{\text {TIS }}$ can be very small (sub-seconds) or even instant. In a real experiment, however, this transition is a process that requires a second-scale time interval of $t_{\text {ramp }}$, i.e. the ramp time of the flow rate onset (Figure 3B). In ACTIS, the runtime is relatively short (peaks of $\mathrm{PL}$ and $\mathrm{L}$ are detected within $30 \mathrm{~s}$ ), i.e. the ramp time of a few seconds may significantly influence TIS of L from PL. Therefore, we varied $t_{\text {ramp }}$ to examine the impact of this variation on the profiles of PL and L and, in turn, on the accuracy of $K_{\mathrm{d}}$ determined with ACTIS. The rest of the parameters were default (see Figure 2B).

\section{SETTING UP A VIRTUAL ACTIS EXPERIMENT IN COMSOL}

To simulate TIS in the five-component setup depicted in Figure 2B we used COMSOL Multiphysics software, version 5.4, with the "Transport of Diluted Species" and "Laminar Flow" modules, which incorporate equations for both mass transfer and reversible binding of $\mathrm{P}$ and $\mathrm{L}$ in an equilibrium mixture.

Computation time depends on the dimensions of the simulated geometries; thus, to reduce this time, the lengths of all the five fluidic components can be scaled down along with the length of the detection window. ${ }^{1}$ The values of $Q_{\text {inj }}$ and $Q_{\text {TIS }}$ must also be scaled down to keep the $l_{2} / Q_{\text {TIS }}$ ratio constant. The scaled down dimensions used in COMSOL computation are reported in SI. Moreover, the mesh size was chosen to minimize computational time and memory requirements. For all the simulations the "finer mesh" setting in COMSOL was used with a computational time of $\approx 3 \mathrm{~h} \quad\left(2 \times\right.$ Intel ${ }^{\circledR} \quad$ Xenon ${ }^{\circledR} \quad$ CPU $\quad$ X5690@3.47 GHz, 96 GB RAM); finer settings such as "extra fine mesh" resulted in some cases in a better accuracy (up to 10 times better); however, they required an order of magnitude longer computational time $(\approx 20 \mathrm{~h})(\mathrm{SI})$.

In all virtual ACTIS experiments, we considered a smallmolecule $\mathrm{L}$ with typical diffusion coefficient of $\mu_{\mathrm{L}}=500 \mu \mathrm{m}^{2} / \mathrm{s}$ and a large $\mathrm{P}$ with a typical diffusion coefficient of $\mu_{\mathrm{P}}=50 \mu \mathrm{m}^{2} / \mathrm{s}$. The remaining parameters used as COMSOL settings were: $k_{\text {on,inp }}=10^{3} \mathrm{M}^{-1} \mathrm{~s}^{-1}, k_{\text {off,inp }}=10^{-3} \mathrm{~s}^{-1}, K_{\mathrm{d}}=k_{\text {off,inp }} / k_{\text {on,inp }}=10^{-6} \mathrm{~s}^{-1}$, $\mu_{\mathrm{PL}}=\mu_{\mathrm{P}}=50 \mu \mathrm{m}^{2} / \mathrm{s},[\mathrm{L}]_{0}=0.5 \mu \mathrm{M}, T=300 \mathrm{~K} .[\mathrm{P}]_{0}$ was varied from $1 \mathrm{nM}$ to $1 \mathrm{mM}$ using 11 different non-zero concentrations plus zero concentration. Three other values of $k_{\text {off,inp }}$ i.e. $10^{-4}, 10^{-5}$, and $10^{-6} \mathrm{~s}^{-1}$ with respective values of $K_{\mathrm{d} \text {,inp }}, 10^{-7}, 10^{-8}$, and $10^{-9}$ $\mathrm{M}$, were also used in COMSOL. Testing several $k_{\text {off }}$ values allowed us to verify the independence of the simulations on a particular value of $k_{\text {off }}$, and, therefore, $K_{\mathrm{d}}$; the simulations were shown to be consistent with the used value of $k_{\text {off,inp }}=10^{-3} \mathrm{~s}^{-1}$ (Figure S1)

The sample plug is fully injected during $t_{\text {inj }} / 2\left(t_{\text {inj }}=24 \mathrm{~s}\right)$. Subsequently, the sample plug is displaced from the entrance of the separation capillary inside the capillary at a distance equal to the plug length during another $t_{\text {inj }} / 2$. This displacement is meant to place the initial plug away from the junction between the loopcapillary connector and the separation capillary; flow disturbance is expected to be the greatest near the junction.

The virtual detector was meant to be at the end of the virtual separation capillary, and virtual detection was performed by averaging concentrations across the capillary within a cylindrical detection window (dimensions: $5 \mathrm{~mm} \times r_{2}$ ). Experimentally, the cumulative signal $S$ from protein-bound $\mathrm{L}$ and unbound $\mathrm{L}$ is obtained by averaging points within a time window around the second-peak maximum, i.e. the diffusive peak (around time $\tau_{\mathrm{L}}$ in Figure 1B). ${ }^{1}$ Using a finite-length time window compensates for noise in experimental data. In this work, we used a finite time window to find the cumulative signal $S$ for all our virtual 
experiments. Unless otherwise stated, for all the separagrams, the position of the time window was chosen by default by selecting the time at which $S_{\mathrm{L}}>S_{\mathrm{PL}}$ and $S_{\mathrm{L}}-S_{\mathrm{PL}}=\max$.

All the five fluidic components were modeled as 2D axisymmetric shapes to further reduce computation time in COMSOL.

To study how the variation in the ramp time of flow onset affects the accuracy of $K_{\mathrm{d}}$, a piecewise function was defined in COMSOL for the linear transition from $Q_{\text {inj }}$ to $Q_{\mathrm{TIS}}$, and the length of this transition time, $t_{\text {ramp }}$, was varied.

To study how the variation in plug shape influences the accuracy of $K_{\mathrm{d}}$, a concentration distribution (eq 11) was defined as an analytical function in COMSOL and, then, varied as explained in the previous section.

\section{RESULTS AND DISCUSSION}

General Considerations. It is difficult to setup a benchmark for $K_{\mathrm{d}}$ accuracy, but taking into account that variations in $K_{\mathrm{d}}$ determined experimentally often reach orders of magnitude, we consider deviation by less than a factor of 1.25 as acceptable. Accordingly, for the purpose of this work, we consider $K_{\mathrm{d}}$ accurate if $0.80<K_{\mathrm{d}} / K_{\mathrm{d} \text {,inp }}<1.25$. We assume that the virtual ACTIS system is stable (this was confirmed by repeating virtual runs); therefore, we do not provide random errors. Note that we do not consider the detector as a source of imprecision in the following consideration because highly precise detectors for different detection methods, such as fluorescence and mass spectrometry, are available. In fact, any detector that can measure a cumulative cross-sectional average signal from $\mathrm{L}$ and PL satisfying eq 5 is suitable for accurate ACTIS measurements provided that it satisfies the following requirements. The detector must have a sufficiently high signal readout speed and a concentration limit of quantitation below $K_{\mathrm{d}}$ values of the studied complexes; the limit of quantification $(\mathrm{LOQ}=10 \times \mathrm{S} / \mathrm{N}$; $\mathrm{N}$ : noise) for $\mathrm{L}$ on the used detector should be at least equal to $[\mathrm{L}]_{0}{ }^{26}$

Accuracy of the Default ACTIS Setup. The virtual fivecomponent ACTIS setup, described in the "Setting up a Virtual ACTIS Experiment" section, was first examined for accuracy under default conditions shown in Figure 2B. A full set of separagrams was calculated and $K_{\mathrm{d}}$ was determined from the respective binding isotherm (Figure 4). We found that $K_{\mathrm{d}}$ determined in this setup deviated from $K_{\mathrm{d} \text {,inp }}$ by a factor of 1.02 . The proven accuracy of the default five-component ACTIS setup opened a route for performing a more stringent accuracy test with variations in (i) the radius of the injection loop while keeping its volume constant, (ii) the radius of the separation capillary, (iii) the shape of the initial (prior to start of TIS) plug of the equilibrium mixture, and (iv) ramp time in the flow rate transfer from $Q_{\text {inj }}$ to $Q_{\text {TIS. }}$

Variation of Injection-Loop Radius. In this examination, $r_{1}$ was varied from 5 to $5000 \mu \mathrm{m}$ (the default value for $r_{1}$ was $50 \mu \mathrm{m})$. This variation in $r_{1}$ corresponded to variation in $r_{1} / r_{2}$ from $0.05(=5 / 100)$ to $50(=5000 / 100)$ for the default value of $r_{2}=100 \mu \mathrm{m}$. To keep the volume of the injection loop constant (which is the purpose of the injection loop), $l_{1}$ was changed accordingly for each $r_{1}: l_{1} \sim 1 / r_{1}^{2}$. The rest of the parameters were default (see Figure 2B).

For each value of $r_{1}$, a full set of separagrams has been computed, and the $K_{\mathrm{d}}$ value was determined from a respective binding isotherm (Figure S2). All determined $K_{\mathrm{d}}$ values were compared to $K_{\mathrm{d} \text {,inp }}$ (Figure $5 \mathrm{~A}$ ). We found that $K_{\mathrm{d}}$ was accurate according to our criteria $\left(0.8<K_{\mathrm{d}} / K_{\mathrm{d} \text {,inp }}<1.25\right)$ for $r_{1} / r_{2} \leq 2.5$ but not for $r_{1} / r_{2} \geq 5$. At $r_{1} / r_{2}=0.25$ we obtained a binding isotherm with anomalous shape (Figures S2) and $K_{\mathrm{d}} / K_{\mathrm{d} \text {,inp }}=1.27$. This anomaly was due to a numerical artifact from meshing in COMSOL; by using a more refined mesh we obtained $K_{\mathrm{d}} / K_{\mathrm{d} \text {,inp }}<1.02$. The improvement in the $K_{\mathrm{d}}$ accuracy by mesh
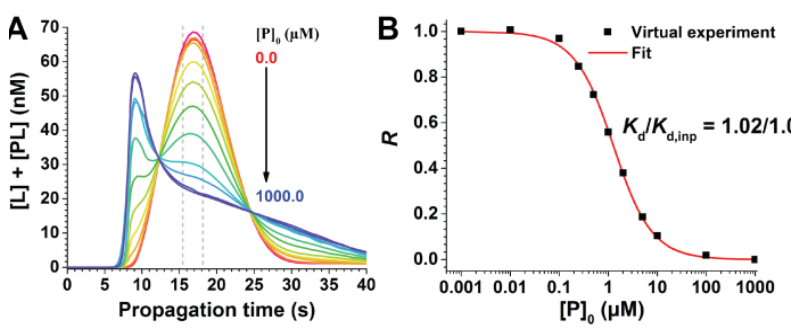

Figure 4. Separagrams (A) and a resulting binding isotherm (B) for a virtual ACTIS experiment in the five-component fluidic system mimicking a realistic ACTIS setup schematically depicted in Figure 2. The dashed lines indicate the time window within which the average signal was taken to calculate the $R_{\exp }$ values using eq 6 . The binding isotherm was fit with eq 7 with $K_{\mathrm{d}}$ as fitting parameter.

refinement may seem to suggest that the large deviation in $K_{\mathrm{d}}$ at $r_{1} / r_{2} \geq 5$ are also due to too coarse of a mesh as well, however a refined mesh at these large ratios did not result in an improvement in $K_{\mathrm{d}}(\mathrm{SI})$.

It is instructive to analyze the shapes of the separagrams (Figure S2) to understand the nature of the large deviations in $K_{\mathrm{d}}$ at $r_{1} / r_{2} \geq 5$. First, for separagrams at $r_{1} / r_{2}$ of up to 2.5 $(=250 / 100)$ they show the two expected peaks - the first for the non-diffusive species (PL) and the second for the diffusive species (L) as in Figure 1B. However, for greater ratios, i.e. $r_{1} / r_{2} \geq 5$, the two peaks merge into one (see shape plots in Figure 5A) followed by a long tail. At these greater ratios, the radius of the injection loop is much larger than that of the separation capillary, and the injection-loop length is very short (Table S1). This large difference between the radii causes the sample plug to take more time to move out of the corner regions of the injection loop that are far removed from the center of the axis (Figure S3) during sample injection. Since the motion of the sample plug out of the injection loop is driven not only by convective motion but also by diffusion, more PL is retained in the injection loop as the sample plug is injected into the separation capillary, since PL diffuses much slowly than L. This retention causes the appearance of a single peak, characteristic of L, followed by a long tail from PL. In summary, $K_{\mathrm{d}}$ could be accurately determined for ratios $r_{1} / r_{2} \leq 2.5$. The ratios $r_{1} / r_{2} \geq 5$ resulted in large deviation in $K_{\mathrm{d}}$; however, it is to be noted that these ratios correspond to $r_{1}>500$ $\mu \mathrm{m}$ and $l_{1}<500 \mu \mathrm{m}$ which are impractical parameters for a physical ACTIS instrument.

Variation in Separation-Capillary Radius. In this examination, the value of $r_{2}$ was varied from 5 to $5000 \mu \mathrm{m}$. Thus, the ratio $r_{2} / r_{1}$ (note the ratio is opposite to $r_{1} / r_{2}$ from the previous study) varied from $0.1(=5 / 50)$ to $100(=5000 / 50)$ for a fixed value of $r_{1}=50 \mu \mathrm{m}$. The rest of parameters were default (Figure 2B). Note that while the ratios between $r_{1}$ and $r_{2}$ change in this and the previous examinations, these two sets of virtual experiments are not identical as decreasing $r_{2}$ while keeping $r_{1}, l_{1}$, and $l_{2}$ constant lead to situations when the plug length is comparable to the length of the separation capillary, which is a greatly suboptimum condition.

For each ratio $r_{2} / r_{1}$, a full set of separagrams has been calculated, and the $K_{\mathrm{d}}$ value was determined from a respective binding isotherm (Figure S4). All determined $K_{\mathrm{d}}$ values were compared to $K_{\text {d,inp }}$ (Figure $5 \mathrm{~B}$ ). $K_{\mathrm{d}}$ values were accurate according to our criteria $\left(0.8<K_{\mathrm{d}} / K_{\mathrm{d} \text {,inp }}<1.25\right)$ for $r_{2} / r_{1} \leq 5 \quad(=250 / 50)$. For $r_{2} / r_{1}>5$, the deviation of $K_{\mathrm{d}}$ from $K_{\mathrm{d} \text {,inp }}$ was beyond the acceptable range: $1.4<K_{\mathrm{d}} / K_{\mathrm{d} \text {,inp }}<6.6$.

Again, it is instructive to analyze the shapes of the separagrams (Figure S4). They show two classical peaks corresponding to the non-diffusive species (PL) and diffusive species (L) (as in Figure 1B) for $r_{2} / r_{1}$ between $1(=50 / 50)$ and 20 $(=1000 / 50)$. For $r_{2} / r_{1}<1$, however, the non-diffusive peak 
becomes less pronounced (see the left shape plot in Figure 5B), which can be attributed to the plug-length $\left(l_{\text {plug }} \geq 52 \mathrm{~cm}\right)$ being greater than the distance from the capillary inlet to the detection window $l_{2}=50 \mathrm{~cm}$ ). Consequently, most of PL and $\mathrm{L}$ in the equilibrium mixture cannot be separated before they reach the detector. Obviously, reducing the plug length by adjusting the injection loop would solve this issue and return these cases to the optimum conditions. Still, $K_{\mathrm{d}}$ deviated from the input $K_{\mathrm{d} \text {,inp }}$ by less than a factor of 1.25 implying that $K_{\mathrm{d}}$ could be accurately determined from these curves.

For $r_{2} / r_{1}>5$, both peaks merged into a single diffusive one (see the right shape plot in Figure 5B). Here, the time of separation $(\geq 500 \mathrm{~s})$ is comparable with or greater than the characteristic time of complex dissociation $\left(\approx 1 / k_{\text {off }}=1000 \mathrm{~s}\right)$; therefore, the injected sample plug is no longer in the state of equilibrium. Moreover, the transfer flow between the small-radius injection loop and the large-radius separation capillary results in the phenomenon of "flow separation". 12 In this situation, the flow is no longer parallel across the whole cross section of the separation capillary. Indeed, recirculation of the flow occurs near the capillary walls (Figure S5) leading to remixing, which affects TIS of L from PL. Additionally, the Péclet number $\mathrm{Pe}$ for large $r_{2}$ $(>500 \mu \mathrm{m})$ becomes smaller than 40 suggesting that longitudinal diffusion along the separation capillary becomes non-negligible at $r_{2} / r_{1}>5\left(P e \approx 42\right.$, Table S1 and S2). ${ }^{13}$ All the above non-optimal conditions combined, i.e. the departure from equilibrium, the "flow separation" phenomenon, and significant longitudinal diffusion, produce a large deviation in $K_{\mathrm{d}}\left(K_{\mathrm{d}} / K_{\mathrm{d} \text {,inp }}>1.25\right)$ at $r_{2} / r_{1}>5$.

In summary, $K_{\mathrm{d}}$ could be accurately determined for $r_{1} / r_{2} \leq 5$. The ratios $r_{1} / r_{2} \geq 10$ resulted in large deviation in $K_{\mathrm{d}}$ due to the different non-optimal conditions described previously. However, if one works within the range of optimal conditions, which correspond to $r_{1} / r_{2} \leq 5$ in our case, it can be concluded that the accuracy of $K_{\mathrm{d}}$ determination by ACTIS is invariant with respect to changes in the separation capillary radius.

Variation in the Shape of the Initial Plug. For this study, the plug shape was varied ranging from an ideal cylindrical shape to conical shapes and to asymmetrical shapes. Here, as opposed to injecting the plug from the injection loop, the initial plug was defined at the entrance of the separation capillary; no injection process with $Q_{\text {inj }}$ was simulated; only the TIS-causing propagation at $Q_{\text {TIS }}$ was computed. The variation in concentration distributions in the initial plug was done by changing parameters $k_{1}$ and $k_{2}$ of the Heaviside function as illustrated in Figure 3A. The rest of the parameters were default (see Figure 2B). For each plug shape, a full set of separagrams has been calculated, and a $K_{\mathrm{d}}$ value was determined from a respective binding isotherm (Figure S6). All determined $K_{\mathrm{d}}$ values were compared to $K_{\mathrm{d} \text {,inp }}$ (Figure $5 \mathrm{C}$ ).

For all examined plug shapes, $K_{\mathrm{d}}$ could be accurately determined, i.e. $0.99 \leq K_{\mathrm{d}} / K_{\mathrm{d} \text {,inp }} \leq 1.08$. The shapes of the separagrams show the two peaks corresponding to the nondiffusive species (PL) and diffusive species (L) (as in Figure 1B) for all plug shapes. All separagrams were only minimally affected by the variations in the plug shape demonstrating that ACTIS was robust towards large variations in the plug shape and proving that moderate imperfections in plug shape expected in real experiments would not affect the accuracy of $K_{\mathrm{d}}$ measurements.

Variation in Ramp Time of Flow Onset. The ramp time $t_{\text {ramp }}$ was varied from 0.10 to $40 \mathrm{~s}$ as illustrated in Figure $2 \mathrm{C}$. The total run time was $60 \mathrm{~s}$ with the diffusive peak (L) arriving to the detector between 15 and $30 \mathrm{~s}$. The rest of the parameters were default (see Figure 2B). For each value of $t_{\text {ramp, a full set of }}$ separagrams has been obtained, and a $K_{\mathrm{d}}$ value was determined from a respective binding isotherm (Figure S7). All determined $K_{\mathrm{d}}$ values were compared to $K_{\mathrm{d} \text {,inp }}$ (Figure 5D). For all examined $t_{\text {ramp }}, K_{\mathrm{d}}$ could be accurately determined, i.e. $0.97 \leq K_{\mathrm{d}} / K_{\mathrm{d} \text {,inp }} \leq 1.17$. The shapes of the separagrams show the two peaks corresponding to the non-diffusive species (PL) and diffusive species (L) (as in Figure 1B) for all $t_{\text {ramp. Overall, }}$. separagram shapes change only slightly; only positions of the non-diffusive and diffusive peaks move from 10 to $25 \mathrm{~s}$ and from 17 to $35 \mathrm{~s}$, respectively (Figure S7). These results show that accuracy of $K_{\mathrm{d}}$ determination with ACTIS is robust towards changes in ramp time of the flow onset.
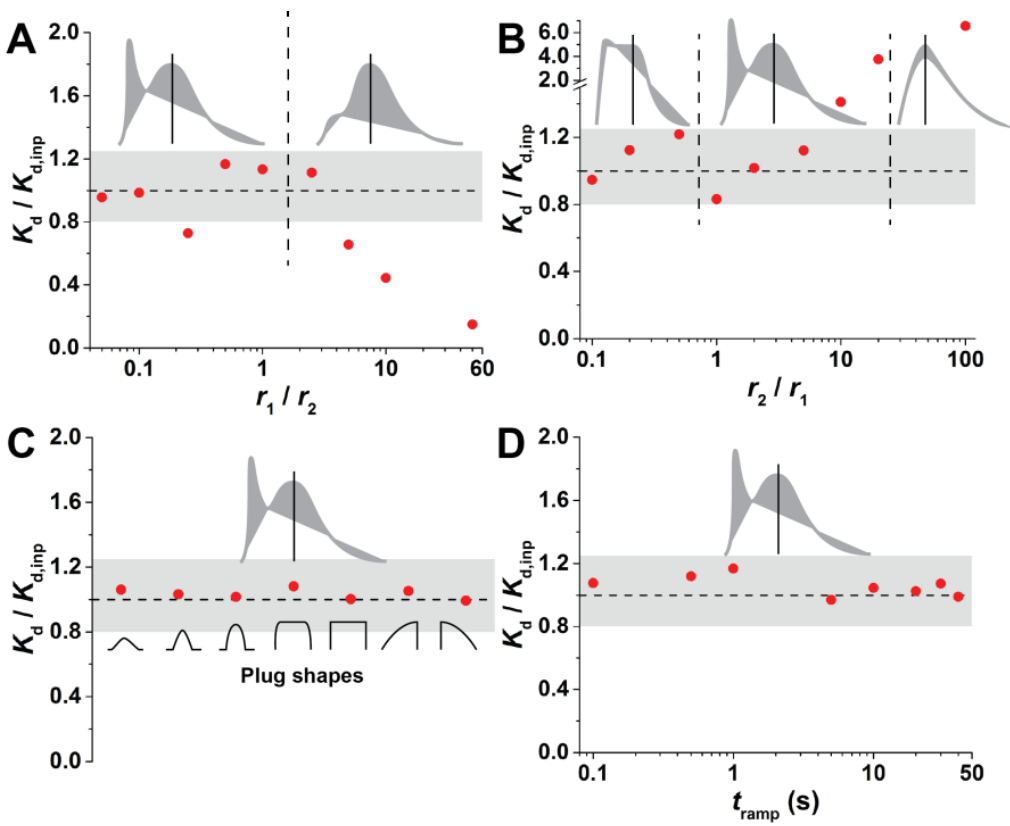

Figure 5. $K_{\mathrm{d}}$ accuracy results of virtual ACTIS experiments by varying: A: injection loop radius $\left(r_{1}\right)$, B: separation capillary radius $\left(r_{2}\right)$, C: injection plug shape, and D: propagation pump ramp time $\left(t_{\text {ramp }}\right)$. The red dots mark the evaluated relative $K_{\mathrm{d}}$ values. The grey area in each panel marks the range in $K_{\mathrm{d}} / K_{\mathrm{d}, \text { inp }}$ (from 0.8 to 1.25 ) which is considered as threshold for accuracy in this study; the horizontal black dashed line marks the target relative $K_{\mathrm{d}}$ value (1.0). The small separagram figures in each panel show the change of shape when varying parameters: black line the window at which the evaluation was done, shaded areas show the areas of change when varying $[\mathrm{P}]_{0}$. Please see the text and supporting information for more details. 


\section{CONCLUSION}

In this work, we examined and proved theoretically the robustness of ACTIS, in a fluidic system resembling the one of a real ACTIS instrument, towards large variations in the parameters characterizing both the fluidic system and the flow. We used COMSOL to construct a virtual five-component ACTIS setup exactly mimicking the geometry of a physical assembly (Figure 2). $K_{\mathrm{d}}$ determined in this setup deviated from $K_{\mathrm{d} \text {,inp }}$, by a factor of only 1.02 .

This five-component ACTIS setup was also used to study how accuracy of $K_{\mathrm{d}}$ is affected by variations in: (i) the radius of the injection loop while keeping its volume constant, (ii) the radius of the separation capillary, (iii) shape of the initial (prior to start of TIS) plug of the equilibrium mixture, and (iv) ramp time in flowrate onset (after the start of TIS). The variations used in the test exceeded markedly the ones that could be caused by undesirable variations expected in a physical ACTIS setup. The tested variations provide wide ranges for desirable experimental instrumental adjustments which would not make ACTIS inaccurate. In general, the values of $K_{\mathrm{d}}$ did not deviate from the input $K_{\mathrm{d}}$ values by more than a factor of 1.25 upon variations in the above parameters. Regarding the variation of the injection loop radius, we found that $K_{\mathrm{d}}$ could be accurately determined for $0.05 \leq r_{1} / r_{2} \leq 2.5$. Larger ratios, i.e. $r_{1} / r_{2} \geq 5$, corresponding to injection loop radii much greater than the separation capillary radius resulted in deviations greater than by a factor of 1.25 . Accuracy of $K_{\mathrm{d}}$ was also not affected greatly by variation of the separation capillary radius if $r_{2} / r_{1}<10$. Note that for $r_{2} / r_{1} \leq 0.5$ the signal variation in the separagram becomes much smaller caused by the plug length being comparable to the separation capillary length (Table S2). A small signal variation will make it difficult to evaluate separagrams containing noise, e.g. from experimental data. Great deviations in $K_{\mathrm{d}}$ were observed for $r_{2} / r_{1} \geq 10$ due the departure from equilibrium of the injected sample plug, the "flow separation" phenomenon, and significant longitudinal diffusion, with deviation in $K_{\mathrm{d}}$ of $K_{\mathrm{d}} / K_{\mathrm{d} \text {,inp }}>>1.25$. Moreover, deviations in plug shape from the ideal cylindrical shape do not influence the accuracy of $K_{\mathrm{d}}$. If plug injection is reproducible from run to run, the determined $K_{\mathrm{d}}$ will be accurate. Finally, large variations in the pump ramp time for flow onset do not influence the accuracy in $K_{\mathrm{d}}$, i.e. cost-effective pumps with slow ramp times can be used for ACTIS.

From the above results and our experience we recommend the following strategy to setup an ACTIS instrument. First, one should choose the propagation flow rate $\left(Q_{\mathrm{TIS}}\right)$ as well as the separation capillary dimensions $\left(r_{2}\right.$ and $\left.l_{2}\right)$ so that the ligand $\mathrm{L}$ reaches the detector at time $\tau_{\mathrm{L}}$ (see eqs. 8-10) which is much shorter than anticipated lifetime of the complex $\left(1 / k_{\text {off }}\right)$. Our choice of $\tau_{\mathrm{L}} \approx 10 \mathrm{~s}$ should suffice most strong complexes. Second, the injection loop radius $r_{1}$ should be selected to be $0.5-1.0$ times the separation capillary radius $r_{2}$. For instance, a separation capillary radius of $100 \mu \mathrm{m}$ corresponds to a range in injection loop radius of 50-100 $\mu \mathrm{m}$. Third, the ACTIS instrument should be assembled using the straightforward scheme provided in Figure 2; an elaborate setup proposed in our preliminary study ${ }^{1}$ is not needed since parameters such as the plug shape or ramp time have only minimal impact on $K_{\mathrm{d}}$ accuracy according to the results of our current study.

Our findings and recommendations will allow ACTISinstrumentation developers to drastically change (simplify) instrument configuration without raising questions about how such changes may affect accuracy of $K_{\mathrm{d}}$. By relying on ACTIS intrinsic accuracy, developers can focus their efforts and resources on optimizing instrument configuration to achieve the highest precision. Finally, to the best of our knowledge, this work provides the first example of a comprehensive proof of accuracy of an analytical method performed in-silico. We foresee that the in-silico accuracy-assessment approach will be used for a similar task on other methods built upon processes with the deterministic nature.

\section{ASSOCIATED CONTENT}

\section{Supporting Information}

The Supporting Information is available free of charge on the ACS Publications website.

Theoretical background for computer simulation and data evaluation; Simulation of separagrams; Figure S1, Variation in $k_{\text {off,inp }}$ - separagrams and binding isotherms; Figure S2, Variation in injection loop dimensions - separagrams and binding isotherms; Figure S3, Variation in injection loop dimensions - sample-plug distribution; Figure S4, Variation in separation capillary radii - separagrams and binding isotherms; Figure S5, Velocity streamlines at different separation capillary radii; Figure S6, Variation in the initial plug shape - separagrams and binding isotherms; Figure S7, Variation in ramp time of flow onset - separagrams and binding isotherms; Table $\mathrm{S} 1+\mathrm{S} 2$, Péclet number and plug length

(PDF)

Evaluation files with raw data (ZIP)

Model files of Numerical simulations in COMSOL (ZIP)

\section{AUTHOR INFORMATION}

\section{Corresponding Author}

*E-mail: skrylov@yorku.ca.

ORCID

Sergey N. Krylov: $\quad 0000-0003-3270-2130$

J.C. Yves LeBlanc: $\quad 0000-0002-3801-3590$

Jean-Luc Rukundo: 0000-0003-3626-2515

Sven Kochmann: 0000-0001-7423-4609

\section{Author Contributions}

The manuscript was written through contributions of all authors, and all authors have given approval to the final version of the manuscript before the submission.

\section{Notes}

The authors declare no competing financial interest.

\section{ACKNOWLEDGMENTS}

This work was supported by the Natural Sciences and Engineering Research Council of Canada (Grant STPG-P521331-2018).

\section{REFERENCES}

(1) Sisavath, N.; Rukundo, J.-L.; Le Blanc, J. C. Y.; Galievsky, V. A.; Bao, J.; Kochmann, S.; Stasheuski, A. S.; Krylov, S. N. Transient Incomplete Separation Facilitates

Finding Accurate Equilibrium Dissociation Constant of ProteinSmall Molecule Complex. Angew. Chem. Int. Ed. 2019, 58, 6635-6639.

(2) Harada, M.; Kido, T.; Masudo, T.; Okada, T. Solute Distribution Coupled with Laminar Flow in Wide-Bore Capillaries: What Can Be Separated without Chemical or Physical Fields? Anal. Sci. 2005, 21, 491-496.

(3) Okada, T.; Harada, M.; Kido, T. Resolution of Small Molecules by Passage through an Open Capillary. Anal. Chem.

2005, 77, 6041-6046. 
(4) Kanoatov, M.; Galievsky, V. A.; Krylova, S. M.; Cherney, L. T.; Jankowski, H. K.; Krylov, S. N. Using Nonequilibrium Capillary Electrophoresis of Equilibrium Mixtures (NECEEM) for Simultaneous Determination of Concentration and Equilibrium Constant. Anal. Chem. 2015, 87, 3099-3106.

(5) Pollard, T. D. A Guide to Simple and Informative Binding Assays. Mol. Biol. Cell 2010, 21, 4057-4298.

(6) Jameson, D. M.; Ross, J. A. Fluorescence

Polarization/Anisotropy in Diagnostics and Imaging. Chem. Rev. 2010, 110, 2685-2708.

(7) Wu, P.; Brand, L. Resonance energy transfer: methods and applications. Anal. Biochem. 1994, 218, 1-13.

(8) Umehara, R.; Harada, M.; Okada, T. Wide-bore hydrodynamic chromatography in sub-second range. J. Sep. Sci. 2009, 32, 472-478.
(9) Taylor, G. I. Dispersion of soluble matter in solvent flowing slowly through a tube. Proc. R. Soc. A: Math. Phys. Eng. Sci. 1953, 219, 186-203.

(10) Umehara, R.; Miyahara, H.; Okino, A.; Harada, M.; Okada, T. Wide-Bore Capillary Hydrodynamic Chromatography with ICP-MS Detection for Evaluation of Lanthanide Uptake by Molecular Aggregates. Anal. Sci. 2012, 28, 359-365.

(11) Kythe, P. K. Sinusoids: Theory and Technological Applications 2014, Chapman and Hall/CRC.

(12) Durst, F. Fluid Mechanics. An Introduction to the Theory of Fluid Flows 2007, Springer.

(13) Chamieh, J.; Leclercq, L.; Martin, M.; Slaoui, S.; Jensen, H., Østergaard, J.; Cottet, H. Limits in Size of Taylor Dispersion Analysis: Representation of the Different Hydrodynamic Regimes and Application to the Size-Characterization of Cubosomes. Anal. Chem. 2017, 89, 13487-13493. 